\title{
Detonation Initiation in a Tube via Imploding Toroidal Shock Waves
}

\author{
S. I. Jackson* and J. E. Shepherd \\ California Institute of Technology, Pasadena, California 91125
}

DOI: $\underline{10.2514 / 1.35569}$

\begin{abstract}
The effectiveness of imploding waves at detonation initiation of stoichiometric ethylene- and propane-oxygennitrogen mixtures in a tube was investigated. Implosions were driven by twice-shocked gas located at the end of a shock tube, and wave strength was varied to determine the critical conditions necessary for initiation as a function of diluent concentration for each fuel. Hydrocarbon-air mixtures were not detonated due to facility limitations, however, detonations were achieved with nitrogen dilutions as large as 60 and $40 \%$ in ethylene and propane mixtures, respectively. The critical-energy input required for detonation of each dilution was then estimated using the unsteady energy equation. Blast-wave initiation theory was reviewed and the effect of tube wall proximity to the blast-wave source was considered. Estimated critical energies were found to scale better with the planar initiation energy than the spherical initiation energy, suggesting that detonation initiation was influenced by wave reflection from the tube walls.
\end{abstract}

\begin{tabular}{|c|c|c|}
\hline$A$ & $=$ & area of annular orifice \\
\hline$A_{h}$ & $=$ & energy scaling variable \\
\hline$A_{\text {tube }}$ & $=$ & cross-sectional area of tube \\
\hline$B_{i}$ & $=$ & constant of integration \\
\hline$c$ & $=$ & speed of sound \\
\hline$D$ & $=$ & Chapman-Jouguet detonation velocity \\
\hline$d$ & $=$ & sensor surface diameter \\
\hline$E_{s}$ & $=$ & source energy \\
\hline$e$ & $=$ & internal energy \\
\hline$\hat{e}$ & $=$ & nondimensional energy \\
\hline$h$ & $=$ & enthalpy \\
\hline$L$ & $=$ & sensor spacing \\
\hline$M$ & $=$ & Mach number \\
\hline$m_{j}$ & $=$ & energy scaling variable \\
\hline$P$ & $=$ & pressure \\
\hline$R$ & $=$ & wave radius or specific gas constant \\
\hline$R_{0}$ & $=$ & explosion length \\
\hline$R_{\sim}$ tube & $=$ & radius of the tube \\
\hline$\tilde{R}$ & $=$ & universal gas constant \\
\hline$\hat{r}$ & $=$ & nondimensional radius \\
\hline$T$ & $=$ & temperature \\
\hline$T_{s}$ & $=$ & postshock temperature \\
\hline$t_{c}$ & $=$ & characteristic time of implosion \\
\hline$U$ & $=$ & velocity \\
\hline$U_{s}$ & $=$ & shock velocity \\
\hline$u$ & $=$ & velocity \\
\hline$u_{r}$ & $=$ & radial inflow velocity through orifice \\
\hline$\hat{u}$ & $=$ & nondimensional velocity \\
\hline$V$ & $=$ & volume \\
\hline$v_{\text {nom }}$ & $=$ & nominal velocity measurement \\
\hline$w$ & $=$ & annular orifice width \\
\hline$\beta_{*}$ & $=$ & ratio of reaction zone length to blast radius \\
\hline$\Delta$ & $=$ & induction length \\
\hline
\end{tabular}

Presented as Paper 3919 at the 40th AIAA/ASME/SAE/ASEE Joint Propulsion Conference and Exhibit, Fort Lauderdale, FL, 11-14 July 2004; received 16 November 2007; revision received 19 March 2008; accepted for publication 27 March 2008. Copyright (C) 2008 by California Institute of Technology. Published by the American Institute of Aeronautics and Astronautics, Inc., with permission. Copies of this paper may be made for personal or internal use, on condition that the copier pay the $\$ 10.00$ per-copy fee to the Copyright Clearance Center, Inc., 222 Rosewood Drive, Danvers, MA 01923; include the code 0001-1452/08 \$10.00 in correspondence with the CCC.

${ }^{*}$ Currently Technical Staff Member, Shock and Detonation Physics, Los Alamos National Laboratory, Los Alamos, NM 87545.

${ }^{\dagger}$ Professor, Aeronautics, MC 105-50. Member AIAA.

$\begin{array}{ll}\Delta t & =\text { transit time } \\ \Delta x & =\text { length } \\ \gamma & =\text { ratio of specific heats } \\ \theta & =\text { reduced activation energy } \\ \lambda & =\text { detonation cell size } \\ \rho & =\text { density } \\ \text { Subscripts } & \\ \text { cylindrical } & =\text { denotes cylindrical wave geometry } \\ h & =\text { geometry coefficient } \\ j & =\text { geometry coefficient } \\ \text { planar } & =\text { denotes planar wave geometry } \\ \text { spherical } & =\text { denotes spherical wave geometry } \\ 0 & =\text { stagnation state } \\ 1 & =\text { initial state } \\ 2 & =\text { state behind shock wave } \\ 5 & =\text { state behind reflected wave } \\ 23 & =\text { average property between transducers } 2 \text { and } 3 \\ * & =\text { critical value required for initiation }\end{array}$

\section{Introduction}

D ETONATION initiation by wave focusing involves propagating an imploding shock or detonation wave into a combustible mixture. The imploding wave geometry forces the shocked gas into an ever-decreasing area that creates additional compression when compared to planar geometries. As the wave radius decreases, the implosion process can develop regions of extremely high postshock pressures and temperatures. The explosion of regions of sufficient size and energy can create a blast wave capable of initiating a detonation wave.

In this study, imploding toroidal shock waves were propagated into a tube containing propane-oxygen-nitrogen and ethyleneoxygen-nitrogen mixtures to test the effectiveness of imploding shock waves at detonation initiation. The energy input used to create the implosion for each test is then estimated and conclusions are drawn as to where detonation initiation occurs in the implosion process based on blast-wave initiation theory.

\section{Previous Research on Imploding Waves}

A large amount of research has been performed with imploding waves in gases. In this study, we briefly review those concerned with the development of the shock implosion facility. More complete reviews on earlier imploding shock and imploding detonation wave works are available elsewhere $[\underline{1}, \underline{2}]$. 
To date, most initiation studies using shock wave focusing have reflected shock waves from both planar and specially shaped end walls to create an imploding, reflected shock wave. The reflection of shock waves from shock tube end walls is a well-established method of initiation $[3,4]$ and is the primary technique used to measure ignition delay times. Several studies have also shown that reflection of a planar incident shock wave from a concave end wall will focus the reflected wave [5-8], and that the temperatures and pressures at the gas-dynamic focus can be sufficient for initiation of the postshock mixture [9-14]. Toroidally imploding shock waves have also been directly initiated from ring sources [15].

Work has been performed with toroidally imploding waves issuing from annular orifices, a feature similar to the current study. Moen et al. [16] and Murray et al. [17] quantified the effectiveness of this geometry on detonation initiation with experiments measuring the transmission of a detonation wave from a smaller-diameter initiator tube to a larger-diameter test section tube. Both tubes were filled with acetylene-air and ethylene-air mixtures, and several different obstacles were placed between the two tubes. When using a circular plate obstacle, they $[16,17]$ noted a substantial increase in the transmission efficiency as compared with cases without obstacles. The annular gap around the obstacle generated an imploding toroidal shock wave downstream, which was trailed by a deflagration. Murray et al. [17] numerically demonstrated that at the focus of this imploding toroid was a region of high-energy density that was responsible for reinitiation of the detonation wave. In hydrocarbonair mixtures, some annular gap sizes allowed successful detonation transmission for tube diameters as much as 2.2 times smaller than cases in which no obstacles were located at the interface. The geometry was less effective for fuel-oxygen mixtures.

In an attempt to improve on this concept, a system was developed $[2,18,19]$ to detonate propane-air mixtures inside a detonation tube using an imploding toroidal detonation wave propagated into the propane-air mixture from the tube walls. To generate the imploding wave, the toroidal initiator used a single spark plug and a small amount of acetylene-oxygen gas. Later simulations [20,21] have shown that the reflection of the primary explosion from the contact surface (separating the gas in the tube from the gas driving the implosion) creates a secondary implosion that is responsible for creation of high pressures and temperatures leading to detonation initiation in this geometry.

The current study is intended to serve as an extension of earlier initiation work using imploding detonation waves [2,17] and is motivated by recent numerical simulations that proposed to further refine this initiation technique through use of an imploding toroidal shock wave (instead of an imploding detonation wave) driven by jets of air or fuel. Li and Kailasanath [22] found that detonations could be initiated in a 14-cm-diam tube filled with stoichiometric ethylene-air using an imploding shock wave created from the injection of a converging annular jet of fuel or air at the outer diameter of the tube. At the injection point, the jet had a Mach number of unity, a pressure of $0.20 \mathrm{MPa}$, and a temperature of $250 \mathrm{~K}$. A total reservoir pressure and temperature of $0.38 \mathrm{MPa}$ and $470 \mathrm{~K}$, respectively, were required to generate such a jet in a perfect gas with $\gamma=1.4$. Detonation initiation via a converging air jet is extremely appealing to designers of pulse detonation engines because it would eliminate the need for a spark plug and associated power supply or any sensitizer fuel. In flight, stagnation of the atmosphere could supply the hot, pressurized air needed to create the imploding wave.

\section{Shock Implosion Facility}

The experimental shock implosion facility was a variation of the classical shock tunnel and consisted of a test section tube with an annular orifice that protruded into the downstream end of a shock tube. It was designed to supply total pressures and temperatures up to $1.68 \mathrm{MPa}$ and $790 \mathrm{~K}$, respectively. These values were in excess of those used for hydrocarbon-air detonation in numerical simulations [22]. This section contains a description of each component of the facility and an overview of its operation.

\section{GALCIT 6 Inch Shock Tube}

The GALCIT (Graduate Aeronautical Laboratories, California Institute of Technology) 6 in. shock tube [23] was used to create the primary shock wave in each experiment and consisted of a $6.20-\mathrm{m}-$ long driver section with a 16.5-cm inner diameter (i.d.) and an 11.3m-long driven section with an i.d. of $15.2 \mathrm{~cm}$. The downstream end flange of the driven section contained a $10.8-\mathrm{cm}$-diam hole through which the upstream end of the test section was inserted into the downstream end of the driven section, as shown in Fig. 1.

Driver and driven sections were separated by a diaphragm held by a hydraulic clamp. The driver section was then pressurized with air, while the driven section pressure remained at atmospheric pressure, causing the diaphragm to bulge into a cutting device [24] located immediately downstream of the diaphragm, which reliably ruptured the diaphragm and caused it to petal open without fragmentation.

The driven section was equipped with four PCB 113A series piezoelectric pressure transducers that were flush-mounted into the tube wall. The transducers, referred to as ST1, ST2, ST3, and ST4, were located at $3.85,0.70,0.20$, and $0.10 \mathrm{~m}$, respectively, from the shock tube end flange. The passage of the shock wave past transducer ST1 triggered the data acquisition (DAQ) system. The two intermediate transducers, ST2 and ST3, were used to record the shock arrival times to calculate the shock velocity. Transducer ST4 provided pressure measurements near the annular orifice on the test section. Data were recorded by a National Instruments data PCIMIO-16E-1 acquisition card running at $250 \mathrm{kHz}$ and processed by a Labview program.

\section{Test Section}

The test section consisted of a 1.0-m-long main tube attached to a shorter $0.25-\mathrm{m}$-long extension tube with an annular orifice. Both components had a constant i.d. of $7.6 \mathrm{~cm}$. The two sections combined to form a tube with an internal length of $1.25 \mathrm{~m}$.

During the experiment, the extension tube was inserted $8.43 \mathrm{~cm}$ into the driven section of the shock tube and fixed in place using four latch clamps. The upstream edge of the annular orifice was located $4.62 \mathrm{~cm}$ downstream of the start of the test section. The orifice was $2.54 \mathrm{~cm}$ wide and was interrupted by four 1.0 -cm-wide support struts. The region of the test section that protruded into the shock tube had an outer diameter of $10.2 \mathrm{~cm}$. Further dimensional information for the orifice region is shown in Fig. 2 .

The test section was equipped with four pressure transducers and nine ionization probes (Fig. 3). Ionization probes were spaced $10.4 \mathrm{~cm}$ apart and the first was located $38.4 \mathrm{~cm}$ from the inner face of the upstream test section flange. Probes were numbered I1-I9 with the probe number increasing with distance from the flange. Pressure transducers TS1, TS2, TS3, and TS4 were located at $27.9 \mathrm{~cm}$, $69.6 \mathrm{~cm}, 1.21 \mathrm{~m}$, and $1.25 \mathrm{~m}$, respectively, from the inner face of the flange. Transducer and ionization probe data were recorded on two National Instruments PCI-6610 DAQ cards running in master-slave configuration and processed with a Labview program. Recording of the test section DAQ system was triggered by the arrival of the incident shock wave at transducer ST4 in the shock tube. The DAQ system for the test section recorded $20 \mathrm{~ms}$ of data at a sampling rate of $2.5 \mathrm{MHz}$

The annular orifice was sealed with $17.8-\mu \mathrm{m}$-thick aluminum foil taped to the orifice. Testing determined that thicker diaphragms did not rupture on all sections of the annular orifice simultaneously, resulting in asymmetrical implosions. The $17.8-\mu \mathrm{m}$-thick foil used was standard Reynolds wrap aluminum foil and allowed all sections

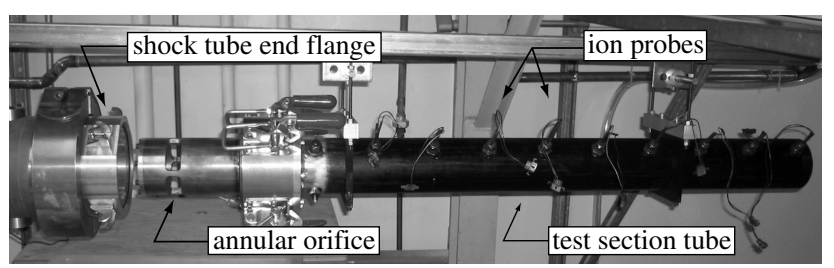

Fig. 1 Experimental setup. 


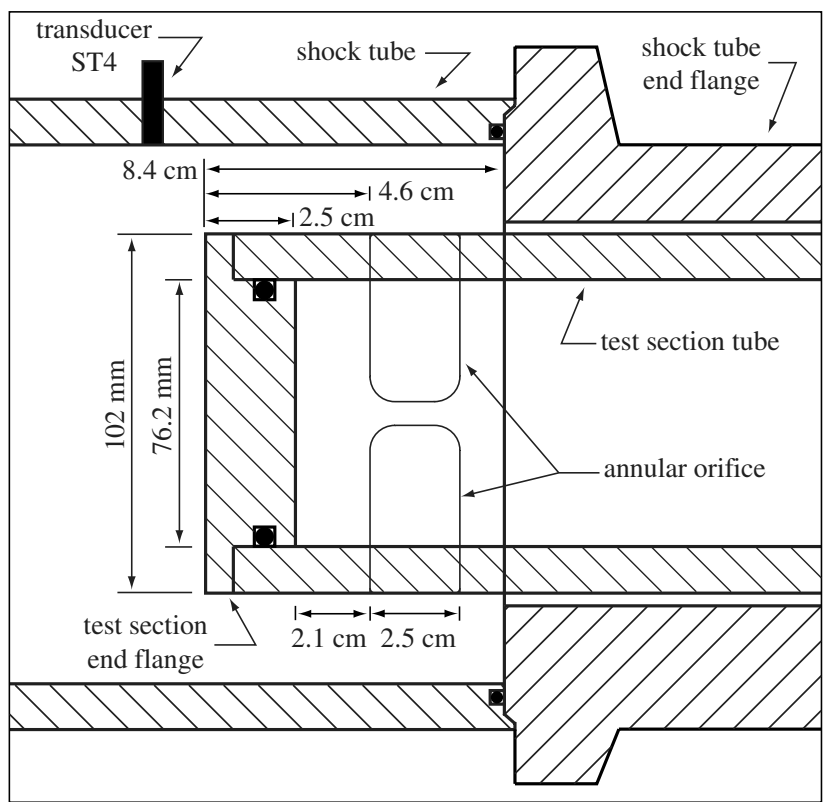

Fig. 2 Dimensional sketch of test section/shock tube interface.

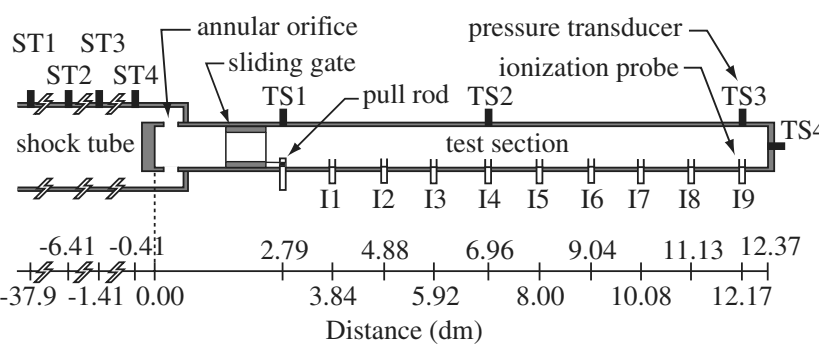

Fig. 3 Dimensional schematic of test section. Measurements are relative to inner face of upstream test section flange. Units are in decimeters $(0.1 \mathrm{~m})$.

of the diaphragm to rupture within $1.5 \mu$ s of each other. For most tests, the diaphragm petaled open cleanly, however, some small fragments were swept into the flow and likely interfered with the implosion symmetry. It is expected that after rupturing the diaphragm, it took some time for the imploding shock to form from the imploding jet of high-pressure gas [25], but this effect was not explored.

The foil provided a reliable seal and rapid rupture but was unable to support more than $0.07 \mathrm{MPa}$ of pressure difference between the test section and the driven section. To prevent premature rupture, a short length of tube with two O-ring seals was inserted into the test section. This sliding gate was used to seal the annular orifice during test section evacuation and filling procedures but was retracted $10 \mathrm{~cm}$ downstream to completely uncover the annular orifice before rupture of the shock tube diaphragm. Gate actuation was enabled via a wire connecting the slider gate to a rotatable pull rod, so that turning the rod pulled the sliding gate along the tube axis. The sliding gate was $8.26 \mathrm{~cm}$ long with an i.d. of $6.35 \mathrm{~cm}$. It is shown removed from the test section in Fig. 4. As the presence of the sliding gate imposed a discontinuous $70 \%$ reduction in the tube area, approximately one tube diameter downstream from the implosion focus, it is possible that it could have induced deflagration-to-detonation transition (DDT) for otherwise marginal cases.

\section{Basic Operation}

During an experiment, the test section slider gate was moved to seal the test section. Diaphragms were placed in the shock tube hydraulic clamp and on the annular orifice of the test section. The test section was secured to the end of the shock tube using the latch

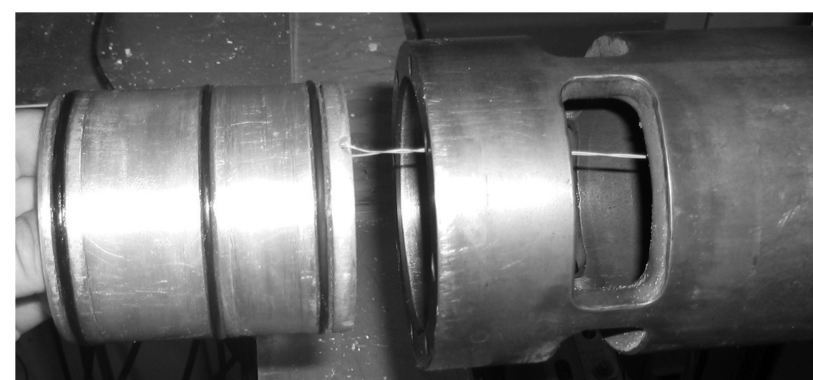

Fig. 4 Sliding gate.

clamps, evacuated, and then filled to $0.10 \mathrm{MPa}$ with the premixed combustible test mixture. Test section gases were premixed for at least 15 min with a brushless fan suspended inside of a 9.25 liter mixture-preparation vessel. The desired composition was achieved by filling the mixture-preparation vessel using the method of partial pressures. After filling, the slider gate was retracted, leaving only the test section diaphragm to separate the test section gas from air in the driven section. Both sections of the shock tube were filled with air at atmospheric pressure. The driver section was then filled with air from a compressed-air source until the shock tube diaphragm burst.

Diaphragm rupture generated a shock wave that traveled downstream in the shock tube and reflected from the upstream flange of the test section, creating a region of slow-moving test gas with elevated pressure and temperature. The increased pressure ruptured the annular orifice diaphragm and created an imploding shock wave in the test section.

\section{Experimental Results and Analysis}

A study of the flow properties at the shock tube/test section interface, including a numerical simulation, was performed to better characterize the initiation process. The results of this analysis are now described, followed by a discussion of each observed initiation mode and summary plots of the initiation data.

\section{Numerical Simulation}

Numerical calculations with AMRITA [26] were conducted to visualize the flow in the test section before initiation. The calculations did not model any chemical reaction or the presence of the diaphragm covering the annular orifice. Figure 5 shows several frames from a simulation where a Mach 1.5 incident wave is initially propagating to the right in the shock tube (frame 1). The simulations are axisymmetric and depict the region shown in Fig. 2. The lower edge of each image is the centerline of the tube. The results indicate that the facility does not create a single implosion inside the test section, but rather a series of closely spaced implosions. The first imploding wave results from the diffracting incident shock wave entering the test section in frame 9. Part of the diffracting wave also reflects from the annular orifice (frame 10), propagating a second imploding wave into the test section. Finally, the diffracting incident shock wave reflects from both the shock tube end flange and the downstream edge of the annular orifice (frame 10). The reflection from the downstream annular orifice then enters the test section, reflecting from the upstream edge of the annular orifice (frame 12) and the axis of symmetry (frame 13). The reflection from the shock tube end flange also enters the test section (frame 13). By the end of the implosion process (frame 17), a standing normal shock wave exists just below the annular orifice, indicating that the flow through the annular orifice is choked.

During experimental testing, an upper bound on the diaphragm rupture time was measured by placing four $127-\mu \mathrm{m}$-diam shielded wires under the diaphragm and across the center of each orifice. One end of each wire was connected to a battery at a $3 \mathrm{~V}$ potential, while the other end was monitored by the DAQ running at $2.0 \mathrm{MHz}$. A shock wave rupturing each panel of the diaphragm would also break each wire, dropping the potential measured at the DAQ end. Wire breakage was assumed to occur when the voltage dropped below 


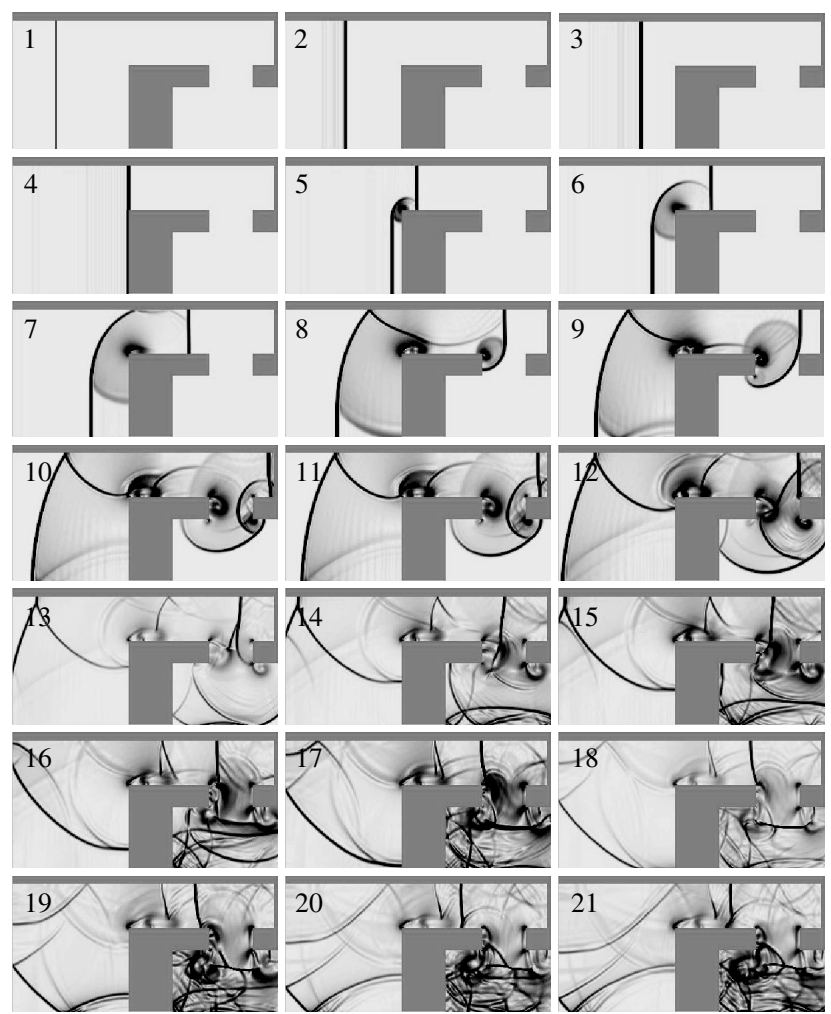

Fig. 5 Pseudoschlieren frames from simulation of shock implosion facility.

$60 \%$ of its initial value. Passage of a Mach 1.5 shock wave resulted in all wires dropping below the $60 \%$ threshold within a $2.0 \mu$ s period, implying near simultaneous rupture of all diaphragm panels. Calculating the shock location vs time from the pressure transducer data shows that wire breakage occurred $100 \mu$ s after arrival of the shock wave over the center of the annular orifice. During that $100 \mu \mathrm{s}$ period, a Mach 1.5 shock wave would only have time to reflect from the shock tube end flange and travel upstream $1.55 \mathrm{~cm}$, placing it $0.25 \mathrm{~cm}$ over the downstream edge of the annular orifice. The break wires used were thicker than the diaphragm material and shielded with a plastic coating that was considerably tougher than the brittle aluminum foil diaphragm. For this reason, it is thought that diaphragm rupture would have occurred before wire breakage and the $100 \mu \mathrm{s}$ break period is considered the maximum time required for diaphragm rupture. Thus, although the diaphragm presence will alter the implosion structure from that shown in the numerical simulation, some type of multiple-implosion structure was also expected in the experiment. Such a shock configuration enhances the possibility for interactions between the two imploding waves to promote initiation, as discussed in Wang et al. [21].

\section{Reflected Wave Pressure}

During each experiment, the Mach number of the incident shock wave was determined from the wave arrival times at pressure transducers ST2 and ST3 in the shock tube.

$$
M_{23}=\frac{\Delta x_{23}}{\Delta t_{23} \sqrt{\gamma_{1} R_{1} T_{1}}}
$$

This allowed the postshock pressure to be determined using the shock-jump equations for a perfect gas and the conditions behind the reflected wave to be estimated by assuming that the flow behind the reflected wave had zero velocity $\left(u_{5}=0\right)$ :

$$
\frac{P_{5}}{P_{1}}=1+2\left(\frac{P_{2}}{P_{1}}-1\right) \frac{1+\{(1 / 2)+[(\gamma-1) /(\gamma+1)]\}\left(M_{23}^{2}-1\right)}{1+[(\gamma-1) /(\gamma+1)]\left(M_{23}^{2}-1\right)}
$$

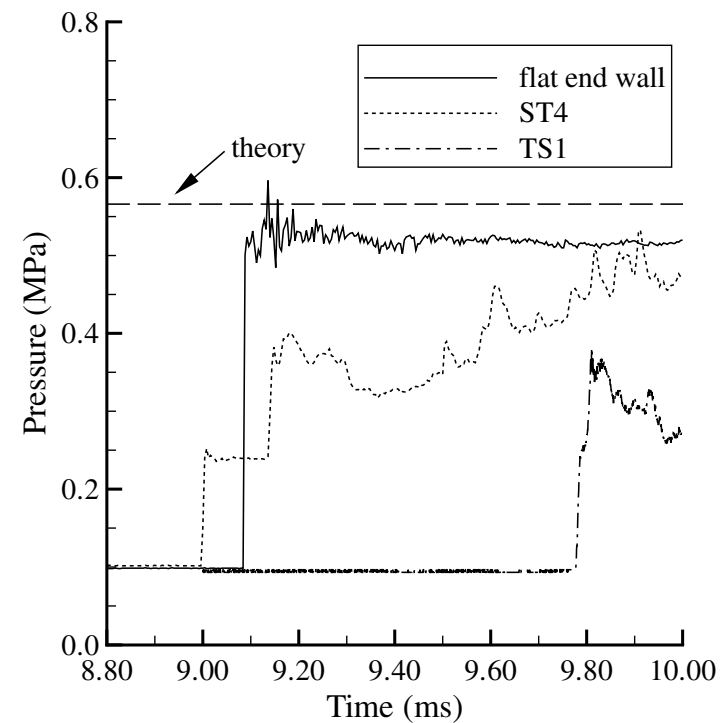

Fig. 6 Postshock pressures for different end wall conditions.

Thus, the pressure ratio across the reflected shock wave can be approximated as a function of the measured incident Mach number. A similar relationship for the reflected temperature can be found with this assumption.

The actual $P_{5}$ values measured in the experiment (at transducer ST4) were initially significantly lower than that predicted by shock tube theory [Eq. (2)]. Experiments were performed to quantify this effect. Figure 6 compares the expected $P_{5}$ value from Eq. (2) to experimental data obtained from a $M=1.52$ incident shock reflecting from both a flat end wall and the test section assembly. Transducer data from the test section assembly (ST4 and TS1) correspond to a test where detonation occurred at a time of $15 \mathrm{~ms}$, which was after wave reflection from the downstream test section end flange. The wave shown in the TS1 trace is a nonreacting shock wave.

For the flat end wall case, Fig. 6 shows the postshock pressure measured at the flat end wall to be about $10 \%$ lower than the expected value, possibly due to heat losses to the wall. With the test section installed, multiple reflections are present in the annular orifice region as shown in the numerical simulations (Fig. 5). Data collected at ST4 shows the first reflection from the upstream test section flange arrives at approximately $9.2 \mathrm{~ms}$ and its postshock pressure is initially about $40 \%$ lower than that expected from a single reflected wave. The second reflection, from the shock tube end flange, eventually reaches ST4 and brings the pressure up to that of the flat wall case, although the simulation shows that it is diffracted both by the complex flow around the upstream test flange and the flow into the annular orifice. The data in Fig. 6 indicate that it takes approximately $0.6 \mathrm{~ms}$ for this wave to reach transducer ST4, although it passes the annular orifice region earlier in time.

\section{Classification of Data}

Each test was classified as one of four outcomes depending on the mode of combustion observed. The four possibilities were prompt detonation, DDT, reflected detonation, and no initiation. Examples of each are presented now as combined pressure-time and space-time diagrams with distance values corresponding to the scale shown in Fig. 3. Zero distance on the vertical axis corresponds to the inner edge of the test section upstream flange. Negative distances are located in the shock tube, whereas positive distances are located in the test section. Pressure trace baselines (dotted lines) indicate the location of the transducer relative to zero distance. The square symbols connected by a dashed line are ionization probe data indicating the arrival of a strong reaction front at the location and time indicated. A wave was considered to be a detonation if the average wave speed in between each pair of ionization probes was within $10 \%$ of the Chapman-Jouguet (CJ) velocity $U_{\mathrm{CJ}}$ and the shock wave was closely 


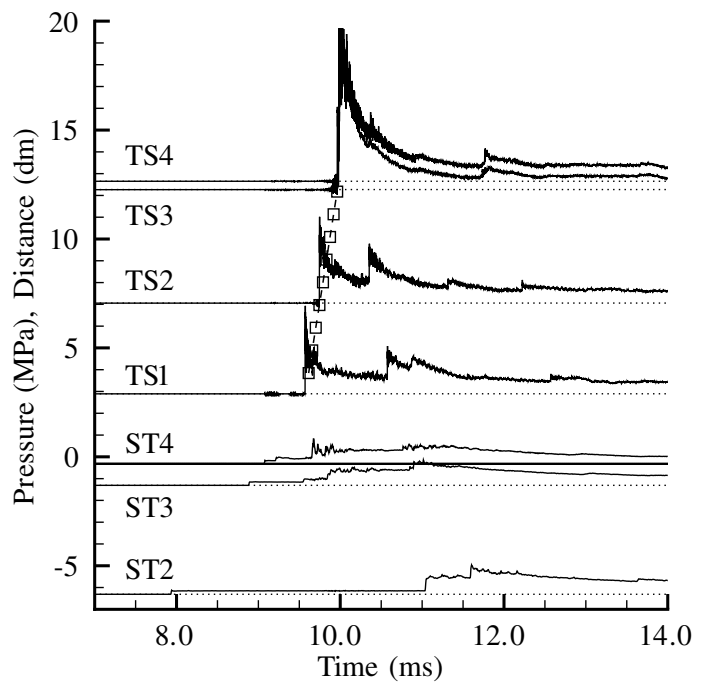

Fig. 7 Prompt detonation initiation.

coupled to the combustion front. Wave overpressures were also examined to ensure the presence of a detonation.

\section{Prompt Detonation}

During a prompt detonation, the first pressure transducer TS1 and ionization probe $\mathrm{I} 1$ in the test section detected a detonation wave. An example of such an outcome in a mixture of $\mathrm{C}_{2} \mathrm{H}_{4}+3 \mathrm{O}_{2}$ is shown in Fig. 7. In the combined pressure-time and space-time diagram, the lower three shock tube pressure traces show propagation of the incident shock wave with a Mach number of 1.52 . Shortly after $9 \mathrm{~ms}$, the incident wave reflects from the annular orifice region and generates an imploding wave in the test section that initiates a detonation. The detonation pressure is recorded by the transducers and the arrival of the reaction zone is detected by the ionization probes. Ionization probe data show that the detonation is propagating at a constant speed within $10 \%$ of $U_{\mathrm{CJ}}$ and coupling between the shock and the reaction front is evident in Fig. 7. Meanwhile, in the shock tube, the reflected shock wave travels back down the tube and is chased by a larger pressure wave from the detonation initiation in the test section. The larger wave eventually overtakes the reflected shock as can be seen in the lowermost pressure trace.

\section{Deflagration-to-Detonation Transition}

Experiments were labeled DDT when the combustion mode was observed to transition from a deflagration to a detonation before the incident shock wave reached the end of the test section. The DDT process is shown in a mixture of $\mathrm{C}_{2} \mathrm{H}_{4}+3 \mathrm{O}_{2}+0.44 \mathrm{~N}_{2}\left(10 \% \mathrm{~N}_{2}\right.$ by volume) in Fig. 8. A shock wave of Mach number 1.88 travels down the shock tube and reflects from the annular orifice region, as in the previous example. However, a detonation is not immediately initiated. The first wave recorded in the test section is a shock wave. The ionization probes indicate that the shock is trailed by an accelerating deflagration. Near the middle of the test section $(0.7 \mathrm{~m})$, transition to an overdriven detonation $(P=7.0 \mathrm{MPa}$, $U=2670 \mathrm{~m} / \mathrm{s}$ ) occurs. The overdriven detonation relaxes as it travels down the test section and has a pressure and wave velocity characteristic of a CJ detonation wave shortly before it reflects off the test section end wall.

\section{Detonation After Reflection}

Some mixtures only initiated behind the reflected wave in the test section. In Fig. 9, a shock wave of Mach number 1.46 in the shock tube generated an implosion in the test section filled with a mixture of $\mathrm{C}_{2} \mathrm{H}_{4}+3 \mathrm{O}_{2}+4 \mathrm{~N}_{2}\left(50 \% \mathrm{~N}_{2}\right.$ by volume $)$ but failed to detonate the mixture. The implosion process propagated a shock wave through the test section, which reflected from the test section end flange. A large explosion occurred near ionization probe $\mathrm{I} 8$ roughly $2 \mathrm{~ms}$ after

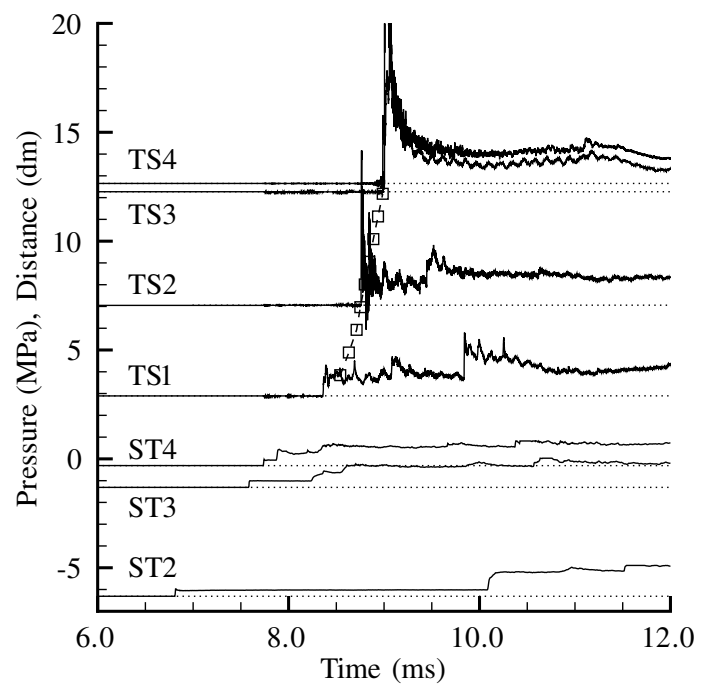

Fig. 8 DDT.

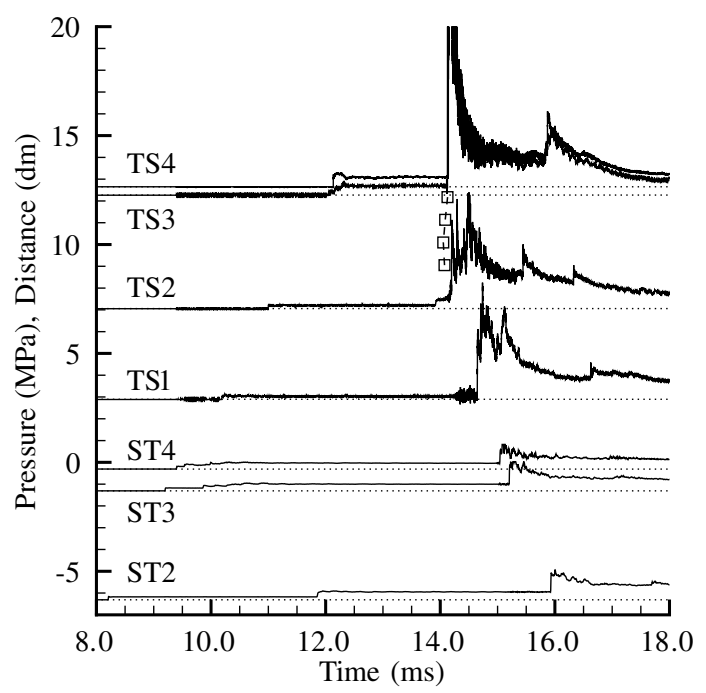

Fig. 9 Initiation behind the reflected wave.

the reflected wave had passed by. The closest pressure trace, TS4, measured the explosion pressure to be $26 \mathrm{MPa}$, which is more than eight times $P_{\mathrm{CJ}}$ for the test mixture. A significant ionization front was detected on only four of the nine transducers in the test section because the increased density behind the incident shock in the test section $(M=1.53)$ compressed the combustible test mixture into the last half of the tube.

\section{No Initiation}

In some cases, low wave speeds and pressures were measured by the pressure transducers and no ionization was detected. Such experiments were deemed initiation failures. Data from such a failure in a mixture of $\mathrm{C}_{2} \mathrm{H}_{4}+3 \mathrm{O}_{2}+6 \mathrm{~N}_{2}\left(60 \% \mathrm{~N}_{2}\right.$ by volume $)$ are shown in Fig. 10. A Mach 1.70 shock wave in the shock tube created a Mach $1 . \overline{75}$ wave in the test section. The wave reflected from the test section end wall and traveled back into the shock tube with no combustion occurring in the $20 \mathrm{~ms}$ data acquisition window.

\section{Initiation Results}

Summary plots of the incident shock tube Mach number $M$ vs percent diluent are shown in Figs. 11 and 12 for stoichiometric ethylene-oxygen and propane-oxygen mixtures with varying nitrogen dilution by volume. During the experiments, the incident shock strength varied from $M=1.31-2.08$. Propagation of an 


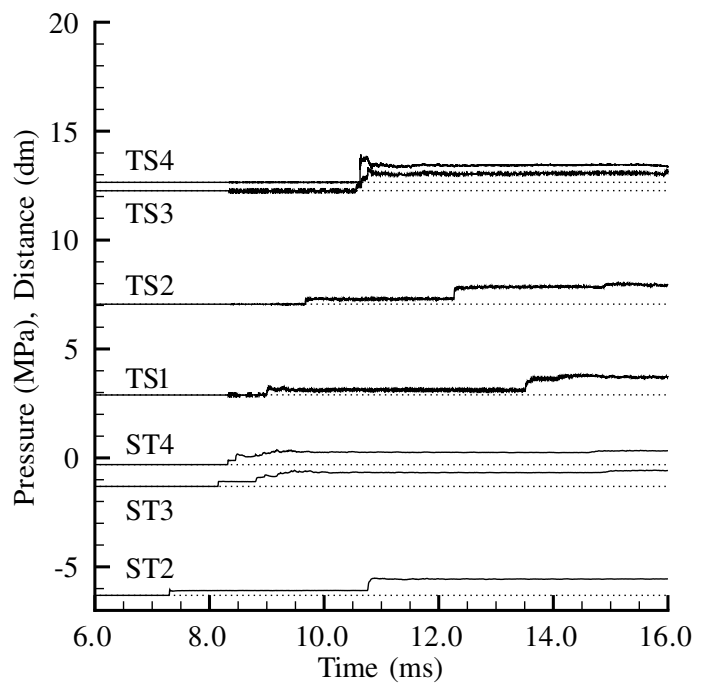

Fig. 10 No initiation.

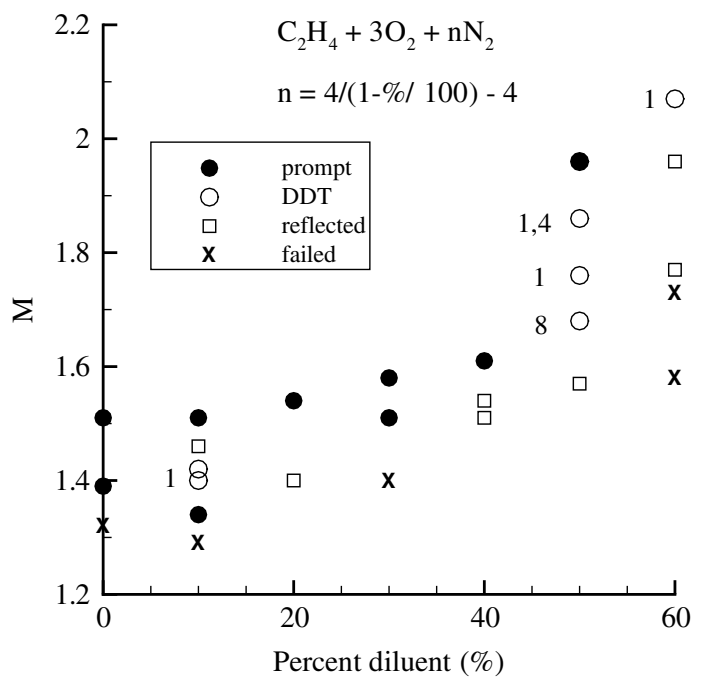

Fig. 11 Initiation results for ethylene-oxygen-nitrogen data.

incident shock Mach number of 2.08 into an atmospheric-pressure test section exceeded the facility limits and was tested only once. Numbers to the left of the DDT data symbols indicate the number of the ionization probe that was closest to the DDT event. Figures 11

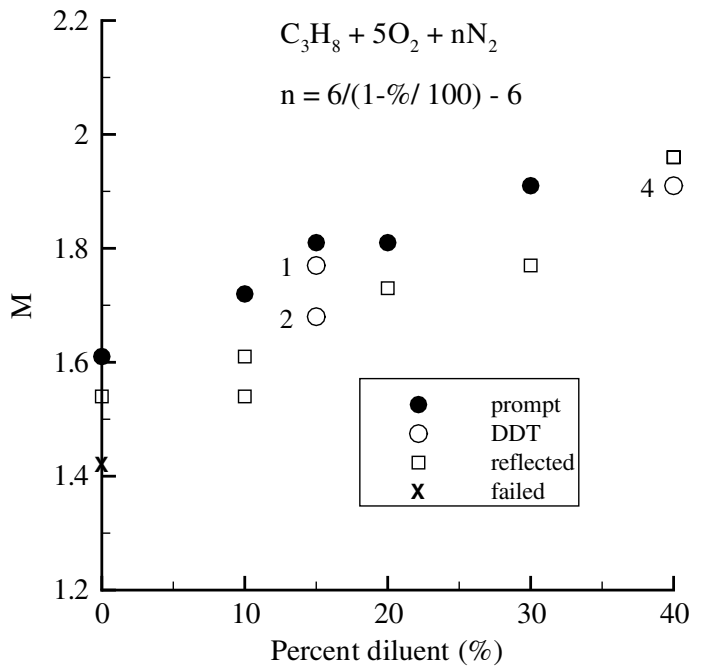

Fig. 12 Initiation results for propane-oxygen-nitrogen data. and 12 show that for a given diluent concentration, a large incident shock strength resulted in prompt detonation in the test section. As $M$ was decreased, prompt detonation no longer occurred, instead resulting in either DDT or detonation initiation behind the reflected shock. If $M$ was too low, combustion no longer occurred.

Both fuels required increasing $M$ to achieve detonation as the amount of dilution was increased. For the ethylene mixtures, this rate of increase jumped dramatically near dilution values of $50 \%$. Propane mixtures exhibited a steeper rate of increase for low dilution values and reached the facility limit at $40 \%$ nitrogen dilution before the presence of a similar trend could be investigated.

Detonation initiation was not achieved for fuel-air mixtures in this study, even when testing at the facility limit of $M=2.08$, which generated a measured reservoir pressure of $1.1 \mathrm{MPa}$. Thus, the experimental data indicate a stronger driver system is required for ethylene-air initiation than was recommended in the numerical work [22]. However, as discussed, the experiment did not create an ideal implosion, but rather several closely spaced implosions whose symmetry was interrupted by the presence of support struts (which blocked $13 \%$ of the annular orifice) and diaphragm fragments. The diameter of the test section was also smaller than in the numerical work [22]. Increasing the diameter of the detonation tube to the values used by $\mathrm{Li}$ and Kailasanath [22] could enhance the initiation process by allowing the imploding shock wave to achieve higher values of compression during the implosion process. However, an increased tube diameter would also reduce the amount of confinement, moving potential reflecting surfaces away from the implosion focus, which has been shown to be detrimental to the success of the implosion process [2]. Thus, it is not clear what net effect changing the diameter would have on the initiation process. Switching to a fuel-air driver gas could also help the process by injecting a combustible mixture into the test section rather than inert air.

\section{Experimental Uncertainty}

The dominant source of uncertainty in the experimental velocity measurements was due to the sampling rate and finite size of the pressure transducers and ion probes used to detect the wave arrival in each test. Wave velocities shown in this work were calculated by dividing the distance $L$ between each transducer by the difference in arrival times $\Delta t$ at each transducer, such that $v_{\text {nom }}=L / \Delta t$. Such a method assumes that waves were centered on each transducer at the instant of data sampling.

The uncertainty associated with this technique can be found by determining the velocity variations that would result from the transducer detecting the wave at its leading and trailing edges, as was done in earlier work [2]

$$
\% \text { velocity error }=\frac{2 d}{L} \times 100 \%
$$

where $d$ is the width of the sensor surface and $L$ is the spacing between sensors. When applied to the current experiment geometry, the error associated with the shock tube velocity measurements was $2.2 \%$ or smaller, whereas the error in the test section ion probe measurements was less than $2.9 \%$. Thus, all velocity measurements for this work are considered accurate to within $3.0 \%$.

As with earlier work [2], it is also possible to estimate the uncertainty in the initial mixtures used in the experiments. The maximum leak rate of the experiment under vacuum was measured to be $20 \mathrm{~Pa} / \mathrm{min}$. After evacuation of the experiment, $5 \mathrm{~min}$ were required to fill the vessel. Thus, a maximum air contamination of $100 \mathrm{~Pa}$ was possible during the filling procedure. Temperature measurements were accurate to within $\pm 3 \mathrm{~K}$. Assuming a worst case compounding of uncertainty for the leak rates and temperature fluctuations, the uncertainty in the wave speed $U_{\mathrm{CJ}}$, as calculated by STANJAN [27], does not exceed $\pm 2 \mathrm{~m} / \mathrm{s}$ corresponding to $0.1 \%$ of the velocity. We do not provide uncertainty values of the energy inflow estimates discussed in latter sections due to the large number of approximations used and the fact that these values are only 
intended to be used to determine the scaling of energy in the experiment.

\section{Critical Energy Considerations}

The shock implosion facility is expected to generate a core of highenergy gas via wave compression. However, it is unclear if that highenergy region then explodes and creates a strong spherical blast wave which transitions to a spherical detonation before reflection from the tube wall, or if wall reflections transform it to a planar blast wave in the tube that then initiates a planar detonation. This section examines which initiation mode is present. First, the energy expected for detonation initiation in planar and spherical geometries based on previous work is reviewed. The energy input to the current experiments is then estimated and used to show that the data scales well with the planar energy mode.

\section{Unconfined Blast-Wave Initiation}

Direct detonation initiation from a blast wave occurs when rapid energy deposition into a mixture (e.g., via an exploding wire or high explosive) generates a strong blast wave that satisfies the condition $\left(U_{s} / c_{0}\right)^{2} \gg 1$. The blast wave immediately begins to decay as it expands; however, for combustible mixtures, sufficiently strong blast waves will evolve into detonation waves [28]. Blast waves that are too weak decay to shock waves trailed by a decoupled deflagration. Early work by Zel'dovich et al. [29] proposed that, in order for the blast wave to successfully transition to a detonation wave, adequate time must be available for the shocked gas to release its chemical energy before the wave decays too much. This led to the understanding that, for successful initiation to occur, the period from the instant of energy release until the blast wave decayed to some minimum value (say $U_{\mathrm{CJ}}$ ) must be on the order of the induction time of the mixture. In terms of chemistry, weak blast waves do not elevate the postshock gas temperature sufficiently long enough for chainbranching reactions to build the necessary radical pool required for a self-sustaining detonation.

Expressing this coupling between the blast-wave decay and the detonation chemistry in terms of length scales, rather than time scales, leads to

$$
\frac{\Delta_{*}}{R_{*}} \leq \beta_{*}
$$

where $R_{*}$ is the wave radius when it has decayed to some critical velocity $U_{*}$, and $\Delta_{*}$ is the mixture-specific induction length for the wave at that velocity. The value $\beta_{*}$ is the critical ratio of reaction zone length to blast-wave radius required for coupling of chemical reactions to the shock wave. If the blast wave decays too rapidly or the mixture's chemical reaction is too slow, the detonation wave will fail to develop.

With this criterion, the nonreactive blast-wave solution [30] can be used to solve for the source energy $E_{s}$. For strong blast waves, the energy contained inside of a spherical control volume bounded by the blast wave is constant,

$$
E_{s}=\rho_{0} U_{*}^{2} R_{*}^{3} \int_{V}\left(\hat{e}+\frac{|\hat{u}|^{2}}{2}\right) \hat{\rho} 4 \pi \hat{r}^{2} \mathrm{~d} \hat{r}=\text { constant }
$$

where the characteristic dimensional parameters of initial gas density $\rho_{0}$, critical radius $R_{*}$, and critical velocity $U_{*}$ have been used to nondimensionalize the integral.

Representing the integral as a constant $B_{1}$ and solving for $R_{*}$ yields

$$
R_{*}=B_{2}\left(\frac{E_{s}}{\rho_{0}}\right)^{1 / 3} U_{*}^{-2 / 3}
$$

where the constant $B_{2}=B_{1}^{-1 / 3}$. Substituting $R_{*}$ into the coupling criterion described by Eq. (4) and solving for $E_{s}$ yields

$$
E_{s} \geq B_{3} \frac{\rho_{0} U_{*}^{2} \Delta_{*}^{3}}{\beta_{*}^{3}}
$$

where the constant $B_{3}=B_{2}^{-3}$. Thus, the minimum energy $E_{\text {spherical }}^{*}$ required for direct initiation of a spherical detonation wave

$$
E_{\text {spherical }}^{*} \propto \frac{\rho_{0} U_{*}^{2} \Delta_{*}^{3}}{\beta_{*}^{3}}
$$

scales with the initial density of the gas and inversely with the cube of the critical ratio $\beta_{*}$. Often, velocities on the order of $U_{\mathrm{CJ}}$ are chosen for $U_{*}$, and either the CJ induction length $\Delta_{\mathrm{CJ}}$ or the cell size $\lambda$ are used for $\Delta_{*}$. The constant of proportionality and critical ratio determined by Zel'dovich et al. [29] predicted lower values by several orders of magnitude than are experimentally observed; however, the cubic dependence of Eq. ( $\underline{8})$ on the length scale was observed in the experimental data.

Subsequently, a number of studies have examined this relationship both experimentally [31,32] and numerically [33]. Comparison of empirical models fitted to the data by Benedick et al. [32] showed agreement with the "surface energy model" [34]:

$$
E_{\text {spherical }}^{*} \cong 430 \rho_{0} D^{2} \lambda^{3}
$$

A theoretical and numerical analysis of blast-wave initiation by Eckett et al. [33] used simplified kinetic models to find a similar expression using induction zone length instead of cell size,

$$
E_{\text {spherical }}^{*} \cong B \rho_{0} U_{*}^{2} \theta^{3} \Delta_{*}^{3}
$$

where their choice for $U_{*}$ was slightly lower than the CJ value. The value $\theta$ is the reduced activation energy

$$
\theta=\frac{E_{a}}{\tilde{R} T_{s}}
$$

in which $\tilde{R}$ is the universal gas constant and $T_{s}$ is the postshock temperature. Eckett et al. [33] showed that this model was in reasonable quantitative agreement with experimental $\mathrm{H}_{2}-$ air, $\mathrm{C}_{2} \mathrm{H}_{4}$ air, and $\mathrm{CH}_{4}-\mathrm{O}_{2}-\mathrm{N}_{2}$ direct initiation data.

To extend Eq. (8) to the planar and cylindrical geometries, it is necessary to consider the dimensions of the $E^{*}$ term. For spherical initiation energies, $E_{\text {spherical }}^{*}$ has dimensions of energy $M L^{2} / T^{2}$. However, for cylindrical initiation, the energy is per unit length, i.e., $E_{\text {cylindrical }}^{*}$ has dimensions of $M L / T^{2}$. Finally, the planar initiation energy $E_{\text {planar }}^{*}$ is per unit area and has dimensions of $M / T^{2}$. To keep Eq. (8) dimensionally correct in these geometries, additional units of length are required such that

$$
E_{h}^{*} R_{*}^{h}=A_{h} \rho_{0} U_{*}^{2} R_{*}^{3}
$$

where $h=2,1$, and 0 for planar, cylindrical, and spherical geometries, respectively. $A_{h}$ accounts for both the constant of proportionality and $1 / \beta_{*}^{3}$ from Eq. ( $\left.\underline{8}\right)$. Collecting terms,

$$
E_{j}^{*}=A_{j} \rho_{0} U_{*}^{2} R_{*}^{j}
$$

where $j=1,2$, and 3 for planar, cylindrical, and spherical geometries, respectively. To date, most comparisons [32,33,35] between theory and experiment have studied only spherical initiation into an unconfined volume, although some work has been done on cylindrical geometries [36,37].

\section{Effect of Confinement on a Blast Wave}

Few studies have been performed to determine the minimum energy required to initiate a detonation from a blast wave inside a tube [38]. This minimum energy $E^{*}$ is expected to be less than $E_{\text {spherical }}^{*}$ due to confinement from the tube walls and greater than the minimum energy $E_{\text {tube }}^{*}$ required for initiation of a planar detonation in the tube. The range between $E_{\text {tube }}^{*}$ and $E_{\text {spherical }}^{*}$ can be several orders of magnitude. For example, in stoichiometric propane-air, the work 
of Radulescu [36] predicts that $E_{\text {spherical }}^{*}=702 \mathrm{~kJ}$ while $E_{\text {tube }}^{*}=$ $E_{\text {planar }}^{*} \times A_{\text {tube }}=0.87 \mathrm{~kJ}$ for a $7.6 \mathrm{~cm}$ i.d. tube.

This large range makes it desirable to bracket the energy more quantitatively. Although actual initiation energies will be designdependent due to inefficiencies associated with each system, it is possible to consider how confining walls near the implosion/ explosion center affect the scaling of $E^{*}$. For example, shock reflection from the tube walls can be responsible for initiation of a detonation kernel, which initiates the detonation wave in the tube. For this situation to occur, the blast wave must be of a sufficient strength such that its reflection from the tube walls generates a region of high-energy density that is capable of starting the detonation kernel. If the blast wave decays too rapidly, or conversely, if the tube diameter is too large, the reflection process will be too weak to have any effect on the detonation initiation. Under these conditions, the critical energy required to initiate a detonation inside the tube can be expected to scale with $E_{\text {spherical }}^{*}$, and the effects of confinement are minimal.

When the effects of confinement are significant, however, the critical energy will be less than $E_{\text {spherical }}^{*}$. It is proposed that the key requirement for successful initiation with confinement is that the blast wave be of a minimum strength $M_{s}^{*}$ when it reflects from the tube wall. At this minimum strength, the reaction front behind the incident wave will decouple and trail the shock front as the wave reflects from the wall. The reflection from the tube wall, initially regular, will transition into a Mach reflection. The Mach stem has been shown to be capable of reinitiation of the detonation wave $[17,39]$, as sketched in Fig. 13. It has been suggested that the reflected shock pressure and temperature must be on the order $P_{\mathrm{CJ}}$ and $T_{\mathrm{CJ}}$ in order for the detonation to succeed. The value of $M_{s}^{*}$ required at the limiting condition is not currently known, and, as such, will be left arbitrary. Thus, the radius of the blast wave when it has decayed to $M_{s}^{*}$ will be denoted by $R_{*}$.

Once the tube walls confine the flow, the blast wave no longer decays spherically, but, instead, will undergo a complex series of reflections and eventually transition to a planar wave propagating axially. If the initiation takes place after the transition to a planar wave, the critical energy will scale with that of the planar case:

$$
\begin{gathered}
E^{*} \propto E_{\text {planar }}^{*} A_{\text {tube }} \quad \text { for } \frac{R_{*}}{R_{\text {tube }}} \geq 1 \\
E^{*} \propto E_{\text {spherical }}^{*} \quad \text { for } \frac{R_{*}}{R_{\text {tube }}} \ll 1
\end{gathered}
$$

Thus, $R_{\text {tube }}$ increases for a given blast-wave energy (and thus, fixed $\left.R^{*}\right), E^{*}$ will transition from Eqs. (14) and (15) (Fig. 14). Thus, confinement enhances the initiation process over only a finite range of tube diameters. Solving for the intersection of $E_{\text {spherical }}^{*}$ and $E_{\text {planar }}^{*} \pi R_{\text {tube }}^{2}$, using the values of Radulescu [36], yields that the transition occurs near $R_{\text {tube }}=21.6 \lambda$, which corresponds to approximately $1.0 \mathrm{~m}$ for propane-air mixtures at $0.10 \mathrm{MPa}$ and $295 \mathrm{~K}$. This logic implies that, for the current study, the initiation energy should scale with the planar energy.

\section{Estimating Experimental Energy Input}

The unsteady energy-balance relation can be used to explore the $E^{*}$ scaling relationship by estimating the energy input to the shock

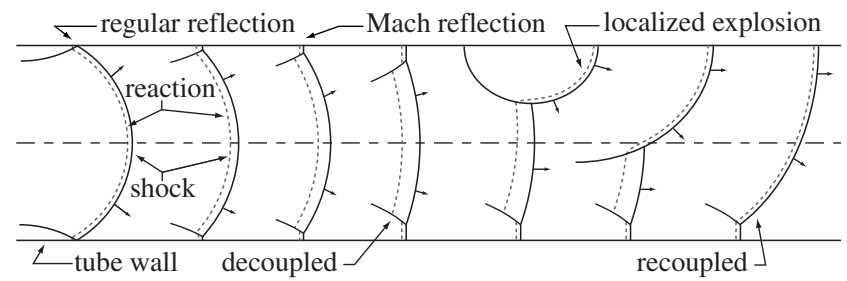

Fig. 13 Reinitiation of the detonation wave from Mach reflection with wall confinement.

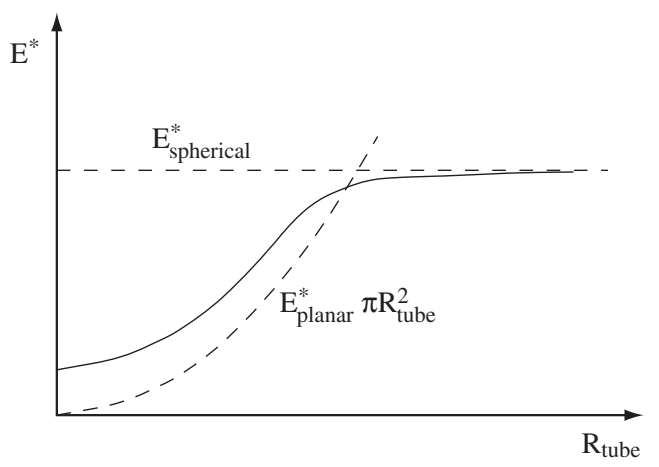

Fig. 14 Critical-energy scaling for a confined blast wave.

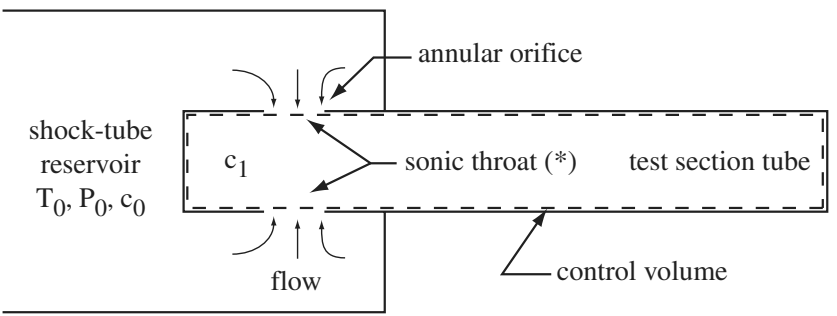

Fig. 15 Control volume considered for shock implosion tests.

implosion initiator. Setting a stationary control volume around the test section tube wall (Fig. 15), and assuming that the flow is adiabatic with no body forces, shear forces, or heat addition, the energy equation is

$$
\frac{\mathrm{d}}{\mathrm{d} t} \int_{V} \rho\left(e+\frac{|u|^{2}}{2}\right) \mathrm{d} V=h_{0} \rho A u_{r}
$$

where $A$ is the area of the annular orifice, $h_{0}$ is the total enthalpy of the inflow (which is conserved), and $u_{r}$ is the velocity of the gas through the orifice. Note that because the control volume follows the inside of the test section wall, all flow must enter the control volume through the annular orifice. The flow velocity $u_{r}$ is assumed to be radially inward and constant across the orifice.

With the assumption that the flow into the orifice behaves as a perfect gas with a constant heat capacity and isentropically chokes at the orifice, the energy input can be expressed as

$$
\int_{V} \rho\left(e+\frac{|u|^{2}}{2}\right) \mathrm{d} V \approx\left(\frac{\gamma}{\gamma-1}\right)\left(\frac{\gamma+1}{2}\right)^{\frac{\gamma+1}{2(1-\gamma)}} P_{0} c_{0}\left(2 \pi R_{\text {tube }} w\right) \frac{R_{\text {tube }}}{c_{1}}
$$

where $2 \pi R_{1} w$ has been substituted for the inflow area $A$. The parameter $w$ is the width of the annular orifice. The equation has been multiplied by a characteristic time $t_{c}$, based on the initial speed of sound $c_{1}$ in the test section and the tube radius, such that $t_{c}=R_{\text {tube }} / c_{1}$.

For an air driver, the value of $\gamma \approx 1.4$ and the energy is approximately

$$
\int_{V} \rho\left(e+\frac{|u|^{2}}{2}\right) \mathrm{d} V \approx 10 w P_{0} R_{\text {tube }}^{2} \frac{c_{0}}{c_{1}}
$$

Figures 16 and 17 show the energy input calculated with Eq. (18) for the data from Figs. 11 and 12. Mach numbers in Figs. 11 and $\overline{12}$ were used with the chemical equilibrium code STANJAN [27] to predict the reservoir conditions $P_{0}$ and $T_{0}$. The data indicate that it takes roughly twice as much energy input to achieve detonation in the propane mixtures compared to the ethylene mixtures.

Two curves are also plotted on each of the figures, which scale with the planar and spherical initiation energies using a form of Eq. (13), 


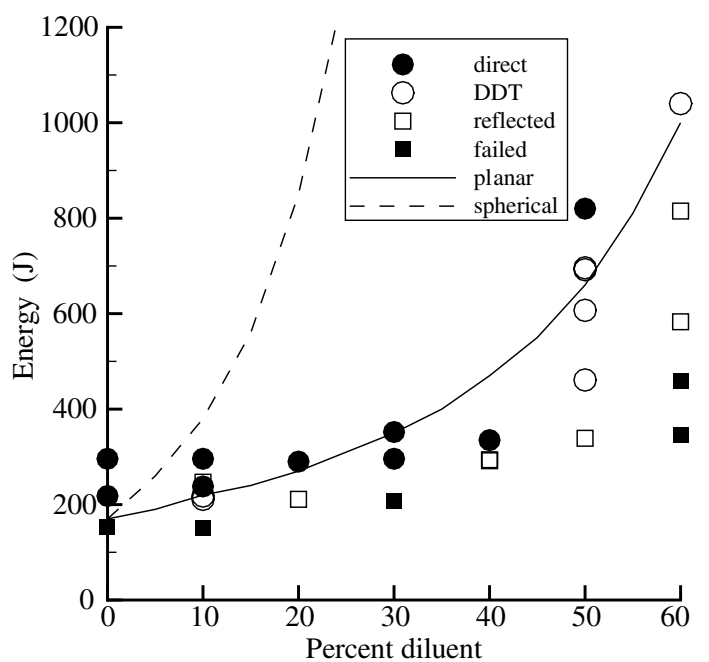

Fig. 16 Energy input to test section tube for shock implosion experiment for ethylene-oxygen-nitrogen data with $m_{1}=0.79$ and $m_{3}=8.3 \times 10^{8}$.

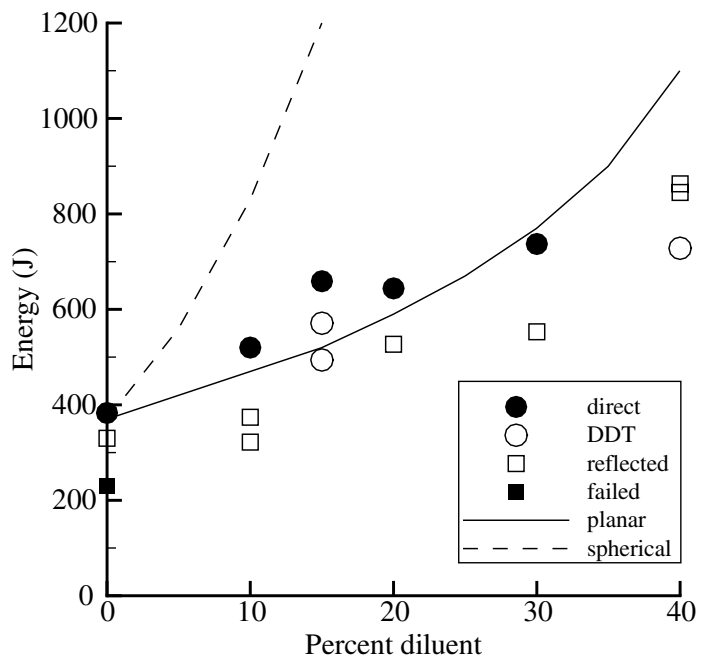

Fig. 17 Energy input to test section tube for shock implosion experiment for propane-oxygen-nitrogen data with $m_{1}=1.6$ and $m_{3}=1.8 \times 10^{9}$.

$$
E_{j}^{*}=m_{j} \rho_{0} D^{2} \Delta^{j}
$$

where $\Delta$ is the mixture-specific induction zone length and $m_{j}$ is a scaling constant that was chosen such that the two curves coincide at $0 \%$ dilution. Induction distances for the scaling curves were computed with the ZND program [40] with the chemical kinetics mechanism of Konnov. are shown in Tables 1 and 2 . Over the range shown, the data agree better with the planar critical-energy trend rather than the spherical critical-energy trend for both the ethylene and propane mixtures. This suggests that initiation is occurring in a planar geometry after wall reflection and that the tube walls are influencing the initiation process.

It is not the intent of this work to present the data of Figs. 16 and 17 as critical energy data for detonation initiation in a tube. Should the assumptions and approximations used to reach Eq. (18) prove valid, the values calculated only represent an upper limit of the energy required for detonation in this configuration, as there was no way to cut off the mass flow (and thus energy input) to the initiator and find the lower limit able to initiate a detonation. Furthermore, theoretical

"Konnov, A., "Detailed Reaction Mechanism for Small Hydrocarbons Combustion, Version 0.4," http://homepages.vub.ac.be/ akonnov, 1998.
Table 1 Ethylene scaling data used in Eq. (19) to generate curve in Fig. 16

\begin{tabular}{lccc}
\hline \hline & \multicolumn{3}{c}{ Ethylene } \\
\cline { 2 - 4 } Diluent & $\rho_{0}, \mathrm{~kg} / \mathrm{m}^{3}$ & $D, \mathrm{~m} / \mathrm{s}$ & $\Delta, \mathrm{mm}$ \\
\hline $0 \%$ & 1.26 & 2376 & 0.031 \\
$10 \%$ & 1.25 & 2317 & 0.041 \\
$20 \%$ & 1.24 & 2259 & 0.054 \\
$30 \%$ & 1.23 & 2198 & 0.075 \\
$40 \%$ & 1.22 & 2132 & 0.107 \\
$50 \%$ & 1.20 & 2060 & 0.164 \\
$60 \%$ & 1.19 & 1977 & 0.282 \\
$70 \%$ & 1.18 & 1874 & 0.617 \\
$73.8 \%$ & 1.17 & 1824 & 0.963 \\
$80 \%$ & 1.17 & 1723 & 2.853 \\
\hline \hline
\end{tabular}

Table 2 Propane scaling data used in Eq. (19) to generate curve in Fig. 17

\begin{tabular}{lccc}
\hline \hline & \multicolumn{3}{c}{ Propane } \\
\cline { 2 - 4 } Diluent & $\rho_{0}, \mathrm{~kg} / \mathrm{m}^{3}$ & $D, \mathrm{~m} / \mathrm{s}$ & $\Delta, \mathrm{mm}$ \\
\hline $0 \%$ & 1.39 & 2361 & 0.030 \\
$10 \%$ & 1.36 & 2306 & 0.040 \\
$20 \%$ & 1.34 & 2252 & 0.054 \\
$30 \%$ & 1.31 & 2195 & 0.076 \\
$40 \%$ & 1.29 & 2132 & 0.114 \\
$50 \%$ & 1.26 & 2062 & 0.187 \\
$60 \%$ & 1.24 & 1981 & 0.358 \\
$70 \%$ & 1.22 & 1879 & 0.916 \\
$73.8 \%$ & 1.20 & 1801 & 2.072 \\
$80 \%$ & 1.19 & 1728 & 4.809 \\
\hline \hline
\end{tabular}

values of $P_{0}$ and $T_{0}$ calculated from the experimentally measured Mach number were used in the calculation, although direct measurements of $P_{0}$ were initially $60 \%$ of the theoretical values. The actual $T_{0}$ is likely lower as well, but was not measured. Accounting for these losses in the calculations with Eq. (18) would significantly decrease the calculated energy input values.

In earlier work, Radulescu et al. [37] determined that the explosion length $R_{0}$, computed from their initiation energies, scaled with the mixture cell size. Such analysis is difficult to justify in the current study because, as mentioned, the energy inputs $E_{s}$ computed in this section overestimate the critical initiation energy. Further complications exist due to the dearth of cell size data in the range of the experiments [41]. With extrapolated cell data and assuming a planar initiation mode for the explosion length, the ratio of the explosion length to the cell size

$$
\frac{R_{0}}{\lambda}=\frac{E_{s}}{P_{0} \lambda}
$$

is approximately 90 and 450 in the current study for ethylene and propane mixtures, respectively. Extrapolation of each case to $\lambda=0$ yields a nonzero value of $R_{0}$. This observation, coupled with the fact that these ratios are typically closer to 20-30 for ethylene-oxygennitrogen and propane-oxygen-nitrogen mixtures, demonstrates that the values of $E_{s}$ in the current study do indeed overpredict the critical initiation energy.

\section{Summary}

Imploding annular shock waves were propagated into a detonation tube filled with ethylene-oxygen-nitrogen and propane-oxygennitrogen mixtures to establish the minimum imploding shock strength necessary to successfully initiate detonations. The minimum shock strength required for initiation was found to increase with increasing dilution. This work is the first experimental verification of the concept of using a nonreacting annular jet to initiate detonation in reactive mixtures and the data indicate that detonation initiation in fuel-air mixtures would require shock driver pressures well above 
those predicted by numerical work [22]. It should be noted, however, that the experiments used a converging jet of air to create the imploding shock wave that was interrupted by support struts and diaphragm fragments. This experimental study also used a smaller diameter tube than the numerical work. These effects prevent a definitive evaluation of the numerical results of $\mathrm{Li}$ and Kailasanath [22], but do provide the best tests to date of this concept.

Criteria predicting the effect of confinement on the scaling of the critical energy for detonation initiation from a blast wave in a tube were reasoned based on a comparison of the decay length of the blast wave vs the radius of the tube walls. The critical-energy input to the shock implosion initiator was estimated for the experimental conditions tested by using the energy equation applied to the test section as a control volume. The critical energy was found to scale better with the energy required to initiate a planar wave rather than a spherical wave, implying that, for the current experiment, detonation initiation was strongly influenced by the tube confinement and occurred after the wave transitioned to a planar front.

\section{Acknowledgments}

This work was supported by the Office of Naval Research (Grant No. N00014-03-1-0931: Detonation Initiation by Annular Jets and Shock Waves) and the NASA Graduate Student Researchers Program (Grant No. NGT8-52937: Initiation System for Pulse Detonation Engines). The authors are grateful to J. Blevins and C. Morris at NASA Marshall Space Flight Center for their support, and would also like to acknowledge discussions on shock implosion with $\mathrm{C}$. $\mathrm{Li}$ and $\mathrm{K}$. Kailasanath. We would like to thank P. Buraczewski for his design work as well as J. Haggerty, B. St. John, and A. Kiani for their machining skills.

\section{References}

[1] Jackson, S., "Gaseous Detonation Initiation via Wave Implosion," Ph.D. Thesis, California Inst. of Technology, Pasadena, CA, 2005.

[2] Jackson, S., and Shepherd, J., "Toroidal Imploding Detonation Wave Initiator for Pulse Detonation Engines," AIAA Journal, Vol. 45, No. 1, 2007, pp. 257-270.

doi: $10.2514 / 1.24662$

[3] Gilbert, R., and Strehlow, R., "Theory of Detonation Initiation Behind Reflected Shock Waves," AIAA Journal, Vol. 4, No. 10, 1966, pp. 1777-1783.

[4] Meyer, J., and Oppenheim, A., "On the Shock-Induced Ignition of Explosive Gases," 13th Symposium on Combustion, Combustion Inst., Pittsburgh, PA, 1971, pp. 1153-1163.

[5] Sturtevant, B., and Kulkarny, V., "Focusing of Weak Shock Waves," Journal of Fluid Mechanics, Vol. 73, No. 4, 1976, pp. 651-671. doi:10.1017/S0022112076001559

[6] Nishida, M., Nakagawa, T., and Kikuyama, Y., "Focusing of Reflected Shock Waves," Transactions of the Japan Society for Aeronautical and Space Sciences, Vol. 28, No. 82, 1986, pp. 209-217.

[7] Nishida, M., "Focusing of Reflected Shock Waves," Proceedings of the International Workshop on Shock Wave Focusing, Tohoku Univ., Sendai, Japan, 1990, pp. 140-153.

[8] Isumi, K., Aso, S., and Nishida, M., "Experimental and Computational Studies Focusing Processes of Shock Waves Reflected from Parabolic Reflectors," Shock Waves, Vol. 3, No. 3, 1994, pp. 213-222. doi:10.1007/BF01414715

[9] Borisov, A., Zamanskii, V., Kosenkov, V., and Lisyanskii, V., "Ignition of Gaseous Combustible Mixtures in Focused Shock Waves," Current Topics in Shock Waves, Vol. 208, AIP Conference Proceedings, American Inst. of Physics, New York, 1989, pp. 696-701.

[10] Chan, C., Lau, D., Thibault, P., and Penrose, J., "Ignition and Detonation Initiation by Shock Focusing," Current Topics in Shock Waves, Vol. 208, AIP Conference Proceedings, American Inst. of Physics, New York, 1989, pp. 161-166.

[11] Gelfand, B., Frolov, S., Medev, S., and Tsyganov, S., "Three Cases of Shock Wave Focusing in a Two-Phase Combustible Medium," Proceedings of the 18th International Symposium on Shock Waves, Springer-Verlag, Berlin, 1992, pp. 831-842.

[12] Gelfand, B., Medvedev, S., Polenov, A., Khomik, S., and Bartenev, A., "Basic Self-Ignition Regimes and Conditions for Their Realization in Combustible Mixtures," Combustion, Explosion, and Shock Waves, Vol. 33, No. 2, 1997, pp. 127-133.
doi:10.1007/BF02671908

[13] Bartenev, A., Khomik, S., Gelfand, B., Gronig, H., and Olivier, H., "Effect of Reflection Type on Detonation Initiation at Shock-Wave Focusing," Shock Waves, Vol. 10, No. 3, 2000, pp. 205-215. doi:10.1007/s001930050008

[14] Gelfand, B., Khomik, S., Bartenev, A., Medvedev, S., Gronig, H., and Olivier, H., "Detonation and Deflagration Initiation at the Focusing of Shock Waves in a Combustible Mixture," Shock Waves, Vol. 10, No. 3 , 2000, pp. 197-204.

doi:10.1007/s001930050007

[15] Barkhudarov, E., Mdivnishvili, M., Sokolov, I., Taktakishvili, M., and Terekhin, V., "Reflection of a Ring Shock Wave from a Rigid Wall," Shock Waves, Vol. 3, No. 4, 1994, pp. 273-278. doi:10.1007/BF01415824

[16] Moen, I., Sulmistras, A., Thomas, G., Bjerketvedt, D., and Thibault, P., "Influence of Cellular Regularity on the Behavior of Gaseous Detonations," Vol. 106, Progress in Astronautics and Aeronautics, AIAA, New York, 1986, pp. 220-243.

[17] Murray, S., Thibault, P., Zhang, F., Bjerketvedt, D., Sulmistras, A., Thomas, G., Jenssen, A., and Moen, I., "Role of Energy Distribution on the Transmission of Detonation," Proceedings of the International Colloquium on Control of Detonation Processes, ELEX-KM Publishing, Moscow, July 2000, pp. 139-162.

[18] Jackson, S., and Shepherd, J., "Initiation Systems for Pulse Detonation Engines," AIAA Paper 2002-3627, July 2002.

[19] Jackson, S., Grunthaner, M., and Shepherd, J., "Wave Implosion as an Initiation Mechanism for Pulse Detonation Engines," AIAA Paper 2003-4280, July 2006.

[20] Yu, S.-T. J., Wang, B., and He, H., "Direct Calculation of Wave Implosion for Detonation Initiation in Pulsed Detonation Engines," Proceedings of the Seventeenth ONR Propulsion Meeting, edited by G. Roy and A. Ghoniem, Office of Naval Research, Arlington, VA, June 2004, pp. 169-180.

[21] Wang, B., He, H., and Yu, S.-T., "Direct Calculation of Wave Implosion for Detonation Initiation," AIAA Journal, Vol. 43, No. 10, 2005, pp. 2157-2169. doi: $10.2514 / 1.11887$

[22] Li, C., and Kailasanath, K., "Detonation Initiation by Annular-JetInduced Imploding Shocks," Journal of Propulsion and Power, Vol. 21, No. 1, 2005, pp. 183-186. doi: $10.2514 / 1.5463$

[23] Smith, J., and Coles, D., "Description of the GALCIT 6" Shock Tube," California Inst. of Technology, Graduate Aeronautical Lab., TR FM67-1, June 1967.

[24] Roshko, A., and Baganoff, D., "Novel Device for Bursting Shock-Tube Diaphragms," Physics of Fluids, Vol. 4, No. 11, 1961, pp. 1445-1446. doi: $10.1063 / 1.1706238$

[25] Murray, S., Zhang, F., Gerrard, K., Guillo, P., and Ripley, R., "Influence of Diaphragm Properties on Shock Wave Transmission," Shock Waves: Proceedings of the 24th International Symposium on Shock Waves, Vol. 2, Springer, Berlin, 2005, pp. 801-806.

[26] Quirk, J., "AMRITA: A Computational Facility (for CFD Modelling)," 29th CFD Lecture Series, Vol. 3, Von Karman Inst., Brussels, 1998.

[27] Reynolds, W. C., "Element Potential Method for Chemical Equilibrium Analysis: Implementation in the Interactive Program STANJAN, Version 3," Technical Rept., Dept. of Mechanical Engineering, Stanford Univ., Stanford, CA, 1986.

[28] Bach, G., Knystautas, R., and Lee, J., "Direct Initiation of Spherical Detonations in Gaseous Explosives," Proceedings of the 12th Symposium (International) on Combustion, Combustion Inst., Pittsburgh, PA, 1968, pp. 853-867.

[29] Zel'dovich, I., Kogarko, S., and Simonov, N., "Experimental Investigation of Spherical Detonation of Gases," Soviet PhysicsTechnical Physics, Vol. 1, No. 8, 1956, pp. 1689-1713.

[30] Landau, L., and Lifshitz, E., Fluid Mechanics, Vol. 6, Course in Theoretical Physics, Translated by J. B. Sykes, and W. H. Reid, Pergamon, London, 1959.

[31] Lee, J., and Matsui, H., "Comparison of the Critical Energies for Direct Initiation of Spherical Detonations in Acetylene-Oxygen Mixtures," Combustion and Flame, Vol. 28, 1977, pp. 61-66. doi:10.1016/0010-2180(77)90008-6

[32] Benedick, W., Guirao, C., Knystautas, R., and Lee, J., "Critical Charge for the Direct Initiation of Detonation in Gaseous Fuel-Air Mixtures," Vol. 106, Progress in Astronautics and Aeronautics, AIAA, New York, 1985, pp. 181-202.

[33] Eckett, C., Quirk, J., and Shepherd, J., "Role of Unsteadiness in Direct Initiation of Gaseous Detonations," Journal of Fluid Mechanics, Vol. 421, 2000, pp. 147-183. doi: $10.1017 / \mathrm{S} 0022112000001555$ 
[34] Lee, J., Knystautas, R., and Guirao, C., Proceedings of the First Specialist Meeting on Fuel-Air Explosions, Univ. of Waterloo Press, Waterloo, Ontario, Canada, 1982.

[35] Vasil'ev, A. A., "Gaseous Fuels and Detonation Hazards," Proceedings of the 28th Fraunhofer ICT Conference on Combustion and Detonation, edited by N. Eisenreih, Fraunhofer Inst. of Chemical Technology, Karksruhe, Germany, June 1997.

[36] Radulescu, M., "Experimental Investigation of Direct Initiation of Quasi-Cylindrical Detonations," M.S. Thesis, McGill Univ., Montreal, 1999.

[37] Radulescu, M., Higgins, A., Murray, S., and Lee, J., "Experimental Investigation of the Direct Initiation of Cylindrical Detonations," Journal of Fluid Mechanics, Vol. 480, 2003, pp. 1-24. doi: $10.1017 / \mathrm{S} 0022112002003154$
[38] Tang, M., Nicholls, J., Lin, Z., Sichel, M., and Kauffman, C., "Direct Initiation of Detonation in a Decane Spray," Vol. 106, Progress in Astronautics and Aeronautics, AIAA, New York, 1986, pp. 474-489.

[39] Brophy, C., Werner, S., and Sinibaldi, J., "Performance Characterization of a Valveless Pulse Detonation Engine," AIAA Paper 20031344, Jan. 2003.

[40] Shepherd, J., "Chemical Kinetics and Cellular Structure of Detonations in Hydrogen Sulfide and Air," Vol. 106, Progress in Astronautics and Aeronautics, AIAA, New York, 1986, pp. 294-320.

[41] Kaneshige, M., and Shepherd, J., "Detonation Database," California Inst. of Technology, Graduate Aeronautical Lab., TR FM97-8, 1997

C. Kaplan Associate Editor 